Article

\title{
Pectosomes and Chitosomes as Delivery Systems for Metronidazole: The One-Pot Preparation Method
}

Toril Andersen ${ }^{1}$, Željka Vanić ${ }^{2}$, Gøril Eide Flaten ${ }^{1}$, Sofia Mattsson ${ }^{3}$, Ingunn Tho ${ }^{1}$ and Nataša Škalko-Basnet ${ }^{1, *}$

1 Drug Transport and Delivery Research Group, Department of Pharmacy, Faculty of Health Sciences, University of Tromsø, Tromsø 9037, Norway; E-Mails: toril.andersen@uit.no (T.A.); goril.flaten@uit.no (G.E.F.); ingunn.tho@uit.no (I.T.)

2 Department of Pharmaceutics, Faculty of Pharmacy and Biochemistry, University of Zagreb, Zagreb 10000, Croatia; E-Mail: zeljka.vanic@pharma.hr

3 Department of Clinical Pharmacology, Umeå University, Umeå SE-90185, Sweden; E-Mail: sofia.mattsson@pharm.umu.se

* Author to whom correspondence should be addressed; E-Mail: natasa.skalko-basnet@uit.no; Tel.: +47-77646640; Fax: +47-77646151.

Received: 16 August 2013; in revised form: 29 August 2013 / Accepted: 4 September 2013 / Published: 6 September 2013

Abstract: Mucoadhesive liposomes offer a potential for improved residence time of liposomal systems targeting contact with mucosal tissues, such as in buccal, oral, colon, and vaginal drug delivery. Most of the currently available methods rely on the coating of preformed liposomes by various mucoadhesive polymers. The aim of this study was to develop novel mucoadhesive system by the one-pot preparation method. The pectin- and chitosan-containing liposomes, namely pectosomes and chitosomes, were prepared by the modified solvent injection method. In order to optimize this novel delivery system, we used pectins and chitosans of both high and low degree of esterification/deacetylation (DE/DD), respectively. Sonication was applied to reduce the original vesicle size. All vesicles were characterized for their size, zeta potential, metronidazole entrapment, and stability. Both pectosomes and chitosomes were found to entrap more metronidazole than conventional plain liposomes. Preliminary data indicate that the polymer is present on the liposomal surface, embedded within inner liposomal bilayers, and entrapped inside the aqueous compartment. The next step in the evaluation of this system is the testing of its mucoadhesiveness. 
Keywords: liposomes; pectosomes; chitosomes; metronidazole; mucoadhesion; polymer-coating; vaginal therapy

\section{Introduction}

Bacterial vaginosis, the most common vaginal infection in women of childbearing age, and is often treated by local administration of metronidazole rather than systemically [1]. However, most of the conventional dosage forms are limited in the retention time at the vaginal site, often failing to achieve intended therapy outcome. Several classes of nanopharmaceuticals have been proposed as a mean to overcome the limitations of conventional dosage forms [2]. Our particular interest was mucoadhesion as a means of prolonging vaginal residence time. The mucoadhesive nanopharmaceuticals could ensure prolonged and intimate contact with the mucus, thus enhancing the delivery of drugs to the underlying tissue. Moreover, nanopharmaceuticals could also provide sustained and controlled drug release [3]. The surface properties of mucoadhesive nanopharmaceuticals play an important role in their retention and delivery capacities once they come in contact with the vaginal mucosa and are, therefore, crucial for successful drug therapy [2].

To prepare mucoadhesive nanopharmaceuticals, we have selected two natural polymers of confirmed mucoadhesiveness, namely chitosan and pectin [4]. Both polymers are available in the different molecular weights and degrees of deacetylation/esterification (DD/DE), which are known to affect their physicochemical properties and may have an impact on their mucoadhesiveness [5,6].

Pectin is a polysaccharide obtained from apple pomace or citrus peel. Its structure and properties vary with the source and the conditions applied during isolation. The acid groups of the galacturonic units can be methoxylated and/or amidated to varying degrees. The degree of esterification (DE) is expressed as a percentage of carboxylic groups that carry an ester and will determine both its physical and chemical properties. Pectin is biodegradable, biocompatible, and non-toxic and is therefore a promising polymer for mucoadhesive drug delivery systems $[7,8]$.

Chitosan is obtained by $n$-deacetylation of chitin; a polysaccharide found in the shells of shrimps, consisting of glucosamine and $N$-acetylglucosamine units. Chitosan interacts well with mucin, one of the principal components of mucus. Its main advantages are conformed biodegradability and biocompatibility, as well as the ability to form gels [5].

Mucoadhesive nano- and micro-pharmaceuticals have been prepared as various delivery systems, such as for example microbeads [9], nanoparticles [10], nanoemulsions [11], and polymer-coated liposomes $[12,13]$. Liposomes have been studied for over 40 years as drug delivery systems for various routes of drug administration, including the vaginal route [2]. The concept of the coating of the liposomal surface with a mucoadhesive polymer has been proposed, relatively early, as a means to increase the retention time on the mucosal surface with a specific target being the intestinal surface [14]. However, most of the currently available methods for the preparation of mucoadhesive liposomes rely on the coating of preformed liposomes by various mucoadhesive polymers $[12,13,15]$.

The aim of the current study was to develop a novel mucoadhesive system that would allow for straightforward and simple preparation procedure, such as, for example, a one-pot preparation method. 
Pectin- and chitosan-containing liposomes were prepared by a modified solvent injection method where the dissolved lipid film was injected into diluted solutions of pectin or chitosan. The original vesicle size was reduced by sonication. To optimize the delivery system, pectins and chitosans, of both high and low degree of esterification/deacetylation (DE/DD), were evaluated. Although our current focus is on the topical vaginal therapy, metronidazole [2-(2-methyl-5-nitro-1H-imidazol-1-yl)ethanol], as model drug, can also be used in oral or buccal therapy. Metronidazole exhibits its antimicrobial activity through reduction of its nitro group and the formation of toxic derivatives, inducing the death of susceptible microorganisms through interacting with DNA. In addition, metronidazole is characterized by a low solubility, both in water and in organic solvents, and is therefore challenging to formulate, particularly in topical formulations [16]. All formulations were characterized with respect to their size, zeta potential, metronidazole entrapment, and stability.

\section{Experimental Section}

\subsection{Materials}

Soy phosphatidylcholine (Lipoid S100, Lipoid GmbH, Ludwigshafen, Germany) was a generous gift by Lipoid $\mathrm{GmbH}$. Chitosan of varying degrees of deacetylation, Fiske-SubbaRow reducer, metronidazole, methanol, $n$-propanol, and phosphorus standard solution were purchased from Sigma Aldrich Inc. (St. Luis, MO, USA). Pectins of varying degree of esterification were the product of Herbstreith \& Fox KG (Neuenbürg, Germany). Ammonium molybdate and peroxide were purchased from Merck KGaA, (Darmstadt, Germany), while sulphuric acid was the provided by May and Baker LTD (Dagenham, England). All other chemicals used in the experiments were of analytical grade.

\subsection{Viscosity of Polymer Solutions}

The viscosity of the aqueous solutions of polymers was measured using a rotational viscometer (Haake Viscotester 7 plus, Thermo Electron GmbH, Karlsruhe, Germany) with a TL 5 spindle. The polymer concentrations of the different polymers were adjusted to the viscosity of the aqueous solution in the range of $0.65 \pm 0.20 \mathrm{mPas}$. Samples were tested in triplicate.

\subsection{Preparation of Liposomes}

Liposomes were prepared by the modified method of solvent injection originally described by Gentine et al. [17]. In brief, Lipoid S100 (SPC, $200 \mathrm{mg}$ ), and $20 \mathrm{mg}$ of metronidazole were dissolved in methanol. The solvent was evaporated using a rotoevaporator system (Büchi rotavapor R-124 with vacuum controller B-721, Büchi Vac V-500, Büchi Labortechnik, Flawil, Switzerland) under a vacuum at $45{ }^{\circ} \mathrm{C}$. The resulting lipid film was redispersed in $100 \mu \mathrm{L}$ of $n$-propanol with a micro-syringe pipette (Hamilton Company, Bonaduz, Switzerland). The dispersion was injected via a needle into $2 \mathrm{~mL}$ of aqueous polymer solution of pectin or chitosan, and stirred for $2 \mathrm{~h}$ at room temperature. Two grades of pectins with different degrees of esterification (35\% and 50\% DE, respectively) were used, as well as the two grades of chitosan with different degrees of deacetylation (77\% and 95\% DD, respectively). The respective polymer concentrations were adjusted to be of a similar viscosity as described above 
(2.2), resulting in the final concentration of $0.50 \%(w / w)$ aqueous solution of each of the two pectins, and $0.05 \%(w / w)$ of chitosan DD $77 \%$ and $0.17 \%$ of chitosan DD $95 \%$ in $0.1 \%(v / v)$ acetic acid, respectively. Plain liposomes of the same lipid composition, and prepared under the same conditions, served as control. We also prepared polymer-coated liposomes (preformed liposomes containing the drug and coated as described in [13] for a comparison, however, due to low metronidazole loading, these data were not included).

\subsection{Vesicle Size Reduction}

The size of liposomes was reduced by the sonication using a Sonics High Ultrasonic Processor (Sigma-Aldrich Chemie GmgH, Steinheim, Germany). The samples were sonicated for either 1 or $2 \min (1+1 \mathrm{~min})$ using an ice bath to prevent heating of the samples. The apparatus was allowed a period of cooling down in between sonication runs of about 2-3 min.

\subsection{Entrapment Efficiency}

To remove unentrapped drug from the polymer-containing liposomal dispersions, chitosomes and pectosomes were dialyzed against distilled water for $4 \mathrm{~h}$ at room temperature (Mw cut off: 12-14,000 Daltons; Medicell International Ltd., London, UK). The volume was adjusted to assure the sinks conditions.

The amount of drug entrapped in the liposomal formulations was determined by UV spectrophotometry (Agilent Technologies, Santa Clara, CA, USA). Liposomal samples were dissolved in methanol and metronidazole content measured at $311 \mathrm{~nm}$. The standard curves of metronidazole in methanol were prepared using the concentrations ranging from 2 to $20 \mu \mathrm{g} / \mathrm{mL}\left(R^{2}=0.9999\right)$.

\subsection{Phosphorus Assay}

The content of phosphatidylcholine (PC) was measured using the modified Bartlett method [18]. In brief, the samples were diluted to appropriate concentration in distilled water and an aliquot $(1 \mathrm{~mL})$ mixed with $0.5 \mathrm{~mL}$ of $10 \mathrm{~N} \mathrm{H}_{2} \mathrm{SO}_{4}$ and heated at $160{ }^{\circ} \mathrm{C}$ for a minimum of $3 \mathrm{~h}$. After the cooling, 2 drops of $30 \%(v / v) \mathrm{H}_{2} \mathrm{O}_{2}$ was added and the mixture heated to $160{ }^{\circ} \mathrm{C}$ for $1.5 \mathrm{~h}$. The ammonium molybdate $(4.6 \mathrm{~mL} ; 0.22 \% \mathrm{v} / \mathrm{v})$ and $0.2 \mathrm{~mL}$ of Fiske-SubbaRow reagent were added after the cooling, mixed, and the mixture was heated for $7 \mathrm{~min}$ at $100{ }^{\circ} \mathrm{C}$. All samples were analyzed by UV spectrophotometry at $830 \mathrm{~nm}$. The phosphorus standard solution was used to prepare a standard curve.

\subsection{Particle Size Analysis}

The particle size distributions of the non-sonicated polymer-containing liposomes were determined by photon correlation spectroscopy (PCS) on Zetasizer 3000HS (Malvern Instruments, Malvern, UK). The measurements were performed at a scattering angle of $90^{\circ}$ and a temperature of $25^{\circ} \mathrm{C}$. The dispersions were diluted with $1 \mathrm{mM} \mathrm{NaCl}$, which was previously filtered through $200 \mathrm{~nm}$ Minisart filters, to achieve a count rate between 100 and $300 \mathrm{kcps}$ [16].

The morphology and particle size distributions (based on the number of particles) of the non-sonicated polymer-containing liposomes were also estimated with the aid of an Olympus 
BH-2 microscope equipped with a computer-controlled image analysis system (Optomax V, Cambridge, UK). In all measurements 1000 particles were examined [19].

After size reduction of the polymer-containing liposomes, the particle size distributions of all liposomal dispersions were measured by PCS using a Submicron particle-sizer (model 370, Nicomp, Santa Barbara, CA, USA). The samples were diluted with filtered ( $0.2 \mu \mathrm{m}$ Millipore filters) distilled water until the appropriate count rate (approximately $250-350 \mathrm{kHz}$ ) and measured in triplicate. The polydispersity index (PI) and the average diameter were used to characterize the samples [20].

\subsection{Zeta Potential}

The zeta potential of the non-sonicated and sonicated polymer-containing liposomes was measured with a Nano ZS (Malvern Instruments Ltd, Worcestershire, UK). All samples were diluted in filtered water until an appropriate concentration was achieved and measured in a measuring cell. All results are presented as the average of at least three independent measurements for each liposome formulation.

\section{9. $p H$ Measurements}

$\mathrm{pH}$ was determined both in the polymer solutions and the dispersion of polymer-containing liposomes in order to elucidate whether, and to what extent, an interaction between polymer and liposomes took place. A change in $\mathrm{pH}$ of the polymer solution after the injection of dissolved lipids may be interpreted as a "loss" of dissolved polymer, i.e., polymer could be entrapped or closely associated with the liposomes. $\mathrm{pH}$ was measured at $22^{\circ} \mathrm{C}$ using a calibrated $\mathrm{pH}$ meter (Metrohm AG, Herisau, Switzerland).

\subsection{Stability Testing}

The stability of the newly developed liposomes was determined after one month of storage in a refrigerator $\left(4^{\circ} \mathrm{C}\right)$. All liposomes were tested for the entrapment (retention of the originally entrapped metronidazole), size distributions, and zeta potential.

\section{Results and Discussion}

\subsection{Viscosity of the Polymer Solutions}

To minimize the effect of different polymer viscosities on the formation of liposomes, the viscosity of each polymer solution was determined and the concentrations were adjusted so that the viscosities of the polymer solutions used in the preparation of liposomes were in a similar range $(0.65 \pm 0.20 \mathrm{mPas})$. An overview of the employed viscosities and the corresponding concentration of the polymers can be seen in Table 1 . 
Table 1. Characteristics of the polymer solutions $(n=3)$.

\begin{tabular}{ccc}
\hline Polymer type & Conc. $(\boldsymbol{\%}, \boldsymbol{w} / \boldsymbol{w})$ & Viscosity (mPas) \\
\hline Pectin (35\% DE) & 0.50 & 0.85 \\
Pectin (50\% DE) & 0.50 & 0.67 \\
Chitosan (77\% DD) & 0.17 & 0.75 \\
Chitosan (95\% DD) & 0.05 & 0.49 \\
\hline
\end{tabular}

\subsection{Entrapment Efficiency}

To achieve a successful local treatment of bacterial vaginosis, it is very important to assure that a sufficient amount of the drug remains at the vaginal site over a required period of time [1]. Any mucoadhesive nanopharmaceutical destined to vaginal administration needs to carry a sufficient quantity of drug and release it in a predictable manner [2]. When designing and preparing the new type of mucoadhesive vesicles, we wanted to simplify the preparation procedure by minimizing the steps required for the preparation of mucoadhesive vesicles, particularly the dilution step involved in the conventional coating. Therefore, as comparison, we originally prepared polymer-coated liposomes by the conventional approach, namely coating of preformed liposomes [13]. However, the encapsulation efficiencies for metronidazole in liposomes were all very low (below 5\%; data not shown), and the subsequent coating of liposomes resulted in further dilution and reduction of the concentration of therapeutically available metronidazole. To prepare mucoadhesive liposomes, containing either pectin or chitosan and carrying sufficient drug load, we developed the new approach and vesicle preparation method, which is a modification of the recently reported solvent injection method [17]. The novelty of our method lies in the procedure based on the addition of the polymer solution prior to the formation of liposomes, resulting in liposome coating in situ. To the best of our knowledge, this is a novel approach in preparing polymer-containingvesicles. In mucoadhesivevesicles, the polymer is expected to be both vesicle-entrapped and surface-available, as some of the polymer will be encapsulated in the aqueous compartments of vesicle. The entrapment of metronidazole (Figure 1) in the different formulations was presented as the amount of drug per lipid, normalized after determination of the lipid amount in each formulation as determined by the phosphor assay. It is known that the vesicle size plays an important role in the ability of nanodelivery system to reach the underlying tissue within vaginal cavity, having a direct impact on the success of the therapy [3]. Therefore, we prepared and compared the potential of the non-sonicated and two types of sonicated mucoadhesive formulations to deliver sufficient amount of metronidazole (Figure 1).

The entrapment in both pectosomes and chitosomes was found to be higher than in the plain liposomes, although not on a significant level, due to a relatively larger SD. The sonication for $1 \mathrm{~min}$ did not lead to loss of originally entrapped metronidazole, however longer sonication ( $2 \mathrm{~min}$ ) resulted in smaller liposomes (Table 2), carrying less of the originally entrapped drug, as expected. It appeared that chitosomes were superior to pectosomes with respect to the entrapment of metronidazole (Figure 1). However, again, the differences were not significant. Interestingly, pectosomes prepared from pectin with $50 \% \mathrm{DE}$ appeared to be the most stable regarding the loss of the originally entrapped metronidazole, a fact which needs to be further evaluated. Chitosomes, on the other hand, seemed to lose more of the originally associated drug during sonication. 
Figure 1. Entrapment efficiency of metronidazole in the different liposomal formulations. Pectosomes (35\% and 50\% DE) are labeled in blue, chitosomes (77\% and 95\% DD) green, and the plain liposomes (PL) in purple $(n=3)$.

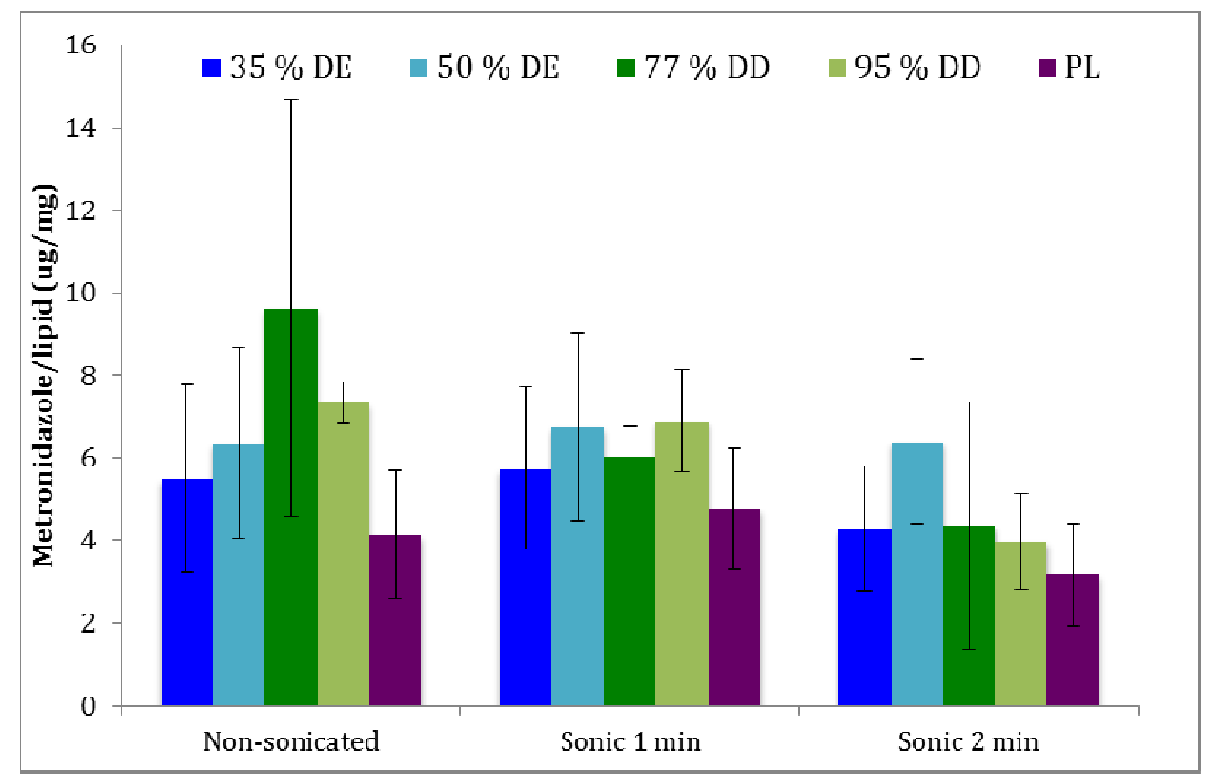

\subsection{Characteristics of Vesicles}

Size determination for non-sonicated vesicles revealed that vesicles were larger than one micron, regardless of their composition or the presence of polymer (data not shown). The polydispersity indexes for those formulations were over 0.70 , therefore, we assumed that mulitilamellar vesicles were formed and their sizes can be taken only as an estimate rather than the absolute values. Although the original isopropanol injection method [17] was developed to manufacture unilamellar vesicles, the presence of polymer onto/into liposomes resulted in larger size. Due to the high polydispersity of vesicle suspension, we applied image analysis to gain a deeper insight on the possible aggregation of the vesicles and their overall structures. However, the image analysis only confirmed that the vesicles were larger than one micron. The vesicle shape and structure was found to be similar for all formulations, although one has to take into the account that image analysis is flattening the $3 \mathrm{D}$ structure and that all vesicles appear spherical. The size and size distributions of sonicated vesicles, both freshly prepared and those stored for one month, are presented in Table 2. The size distributions are expressed as NICOMP distributions, i.e., a bimodal distribution where particles of similar size are grouped in populations and presented as the percentage of particles with the specific mean diameter [20]. The sonication of all types of vesicles resulted in vesicles of smaller size. A very interesting phenomenon has been observed with pectosomes prepared from pectin with $50 \% \mathrm{DE}$; in this case, longer sonication did not result in smaller vesicles, as observed for the other types of liposomes. Those vesicles were also defined by the minimum loss of the originally incorporated metronidazole (Figure 1), which indicates that this pectin grade stabilizes the vesicles and protects the original vesicle structure during the stress caused by sonication. It would be very interesting to explore these vesicles further and we are currently working on their detailed characterization. Chitosomes prepared from $77 \%$ DD chitosan were the smallest of the polymer-coated vesicles (Table 2). Those 
vesicles also lost more of the originally incorporated drug during the sonication process than the other vesicles (Figure 1).

Table 2. Vesicle sizes $(n=3)$.

\begin{tabular}{|c|c|c|c|c|c|c|}
\hline \multirow{2}{*}{ Type of liposomes } & \multirow{2}{*}{$\begin{array}{c}\text { Sonication } \\
\text { time }\end{array}$} & \multicolumn{2}{|c|}{ Peak 1} & \multicolumn{2}{|c|}{ Peak 2} & \multirow{2}{*}{ PI } \\
\hline & & Size (nm) & $\%$ & Size (nm) & $\%$ & \\
\hline \multicolumn{7}{|l|}{ Freshly prepared } \\
\hline Pectosomes & $1 \mathrm{~min}$ & 148 & 13 & 626 & 86 & 0.373 \\
\hline$(35 \% \mathrm{DE})$ & $2 \min$ & 91 & 14 & 324 & 84 & 0.287 \\
\hline Pectosomes & $1 \mathrm{~min}$ & 222 & 11 & 847 & 82 & 0.446 \\
\hline$(50 \% \mathrm{DE})$ & $2 \mathrm{~min}$ & 166 & 12 & 718 & 86 & 0.397 \\
\hline Chitosomes & $1 \mathrm{~min}$ & 62 & 30 & 239 & 72 & 0.315 \\
\hline$(77 \%$ DD) & $2 \min$ & 58 & 21 & 193 & 76 & 0.384 \\
\hline Chitosomes & $1 \mathrm{~min}$ & 194 & 14 & 733 & 86 & 0.442 \\
\hline (95\% DD) & $2 \min$ & 67 & 10 & 290 & 91 & 0.421 \\
\hline \multirow{2}{*}{ Plain } & $1 \mathrm{~min}$ & 91 & 18 & 450 & 83 & 0.329 \\
\hline & $2 \mathrm{~min}$ & 82 & 12 & 415 & 89 & 0.446 \\
\hline \multicolumn{7}{|l|}{ Stored for 1 month } \\
\hline Pectosomes & $1 \mathrm{~min}$ & 115 & 14 & 497 & 85 & 0.324 \\
\hline$(35 \% \mathrm{DE})$ & $2 \min$ & 69 & 14 & 265 & 85 & 0.275 \\
\hline Pectosomes & $1 \mathrm{~min}$ & 113 & 10 & 508 & 90 & 0.390 \\
\hline$(50 \% \mathrm{DE})$ & $2 \min$ & 126 & 16 & 473 & 83 & 0.347 \\
\hline Chitosomes & $1 \mathrm{~min}$ & 68 & 23 & 310 & 75 & 0.360 \\
\hline (77\% DD) & $2 \min$ & 122 & 29 & 410 & 69 & 0.320 \\
\hline Chitosomes & $1 \mathrm{~min}$ & 115 & 6 & 625 & 93 & 0.525 \\
\hline (95\% DD) & $2 \mathrm{~min}$ & 108 & 3 & 774 & 98 & 0.217 \\
\hline \multirow{2}{*}{ Plain } & $1 \mathrm{~min}$ & 69 & 15 & 316 & 85 & 0.367 \\
\hline & $2 \mathrm{~min}$ & 47 & 7 & 222 & 93 & 0.454 \\
\hline
\end{tabular}

Regarding the stability of vesicles with respect to their size distributions, stored vesicles appeared to be smaller than the freshly prepared vesicles. Although contradictory at first glance, we have observed similar behavior with curcumin-containing vesicles [21]; this could be explained by the stabilization of vesicles during the storage at cold temperature and the fact that the measurements of freshly prepared vesicles are actually overestimates and are including agglomerates rather than separated vesicles. The stored chitosomes sonicated for 2 min appear to aggregate and exhibit larger mean diameters than freshly prepared liposomes of the same type (Table 2), which may be contributed to possible loss of the protecting polymer layer on the vesicle surface during sonication and the consequent changes in zeta potential (Figure 2).

The zeta potential of the polymer-containing liposomes and plain liposomes has been determined to provide information on the changes in vesicles surface charge due to the presence of polymer onto/into vesicles (Figure 2). It is evident that the zeta potential of polymer-containing liposomes is changed as a result of the sonication, which could be explained as a consequence of a removal of the charged polymer, from the vesicle outer bilayer, as confirmed for pectosomes. However, in the case of chitosomes, we need to further evaluate the effect of sonication on the change in vesicle surface 
charge. The change in zeta potential is also an indirect indication that polymer was present on the vesicle surface and that less of a polymer is exposed on the outer layers in the sonicated vesicles as compared to the non-sonicated vesicles. The lipid used, SPC, is a neutral lipid, therefore the zeta potential of polymer-containing vesicles can be attributed to the charged polymers.

Figure 2. Zeta potential of different liposomes in relation to applied sonication time. PL, plain liposomes; pectosomes (35\% and 50\% DE); chitosomes (77\% and 95\% DD) $(n=3)$.

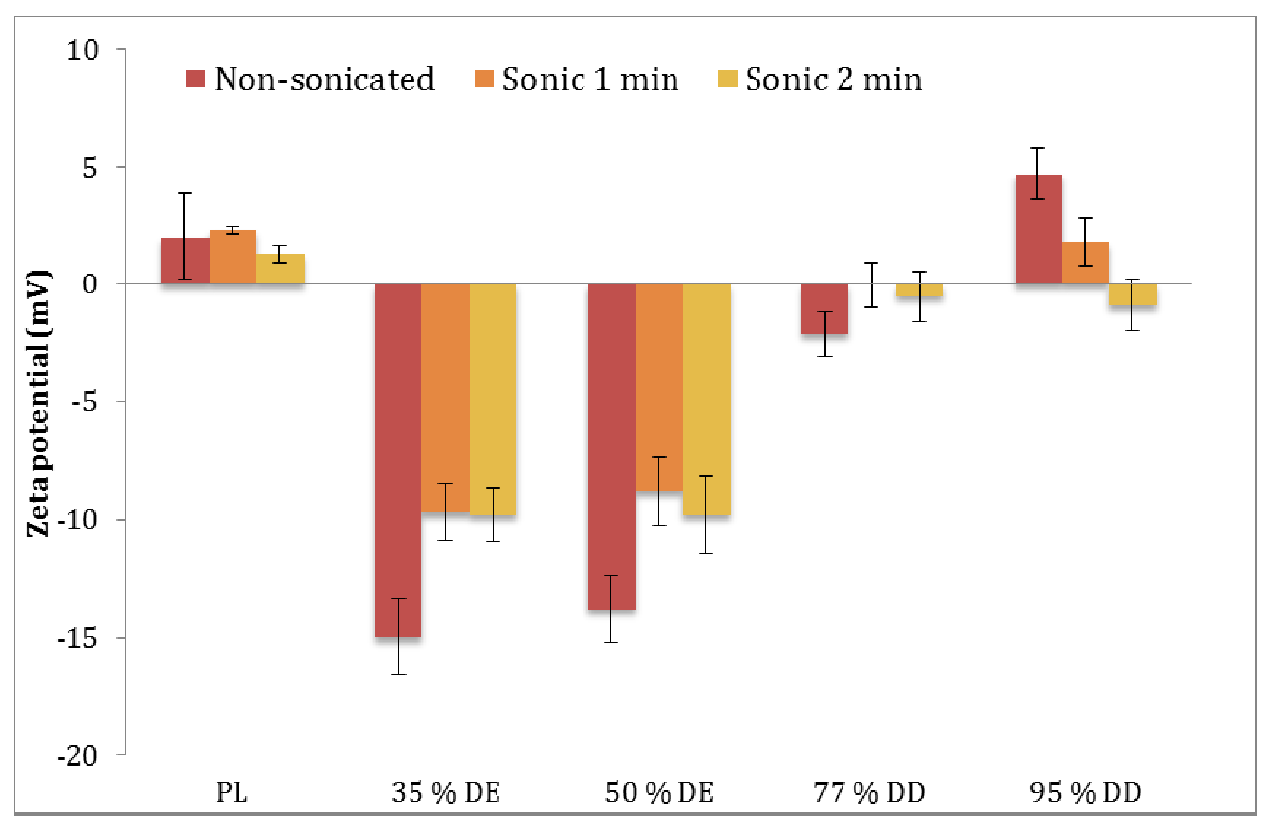

Additional evidence of the presence of polymer on/inside liposomes can be seen through the comparison of the $\mathrm{pH}$ values of polymer solutions versus the $\mathrm{pH}$ of the polymer-containing liposomal dispersions. Whereas the pectin solutions had $\mathrm{pH}$ values of $2.93(35 \% \mathrm{DE})$ and $3.05(50 \% \mathrm{DE})$, respectively, the $\mathrm{pH}$ of pectosome suspensions was $3.31(35 \% \mathrm{DE})$ and $3.42(50 \% \mathrm{DE})$, respectively. Similarly, the $\mathrm{pH}$ of chitosan solutions was 3.43 (77\% DD) and 3.78 (95\% DD), respectively, and chitosomal suspensions exhibited the $\mathrm{pH}$ of 3.91 (77\% DD) and 4.26 (95\% DD), respectively. All polymer-containing vesicle suspensions had a lower $\mathrm{pH}$ than the $\mathrm{pH}$ of the plain liposomal suspension, which was close to $\mathrm{pH}$ of 5.5. The increase in $\mathrm{pH}$ observed in the pectin solutions upon formation of pectosomes might also be taken as a proof that the polymer is also embedded in the vesicles and not only surface-available.

\subsection{Storage Stability}

Figure 3 represents the drug retention values for the liposomally-associated metronidazole after storage for one month at $4{ }^{\circ} \mathrm{C}$. The corresponding vesicle size distributions are presented in Table 2 . Although polymer-containing liposomes retained more of the originally incorporated metronidazole, the differences were not on a significant level. 
Figure 3. Liposomally-retained metronidazole upon storage for one month at $4{ }^{\circ} \mathrm{C}$. Pectosomes $(35 \%$ and $50 \% \mathrm{DE})$ are labeled in blue, chitosomes $(77 \%$ and $95 \% \mathrm{DD})$ green, and the plain liposomes (PL) in purple $(n=3)$.

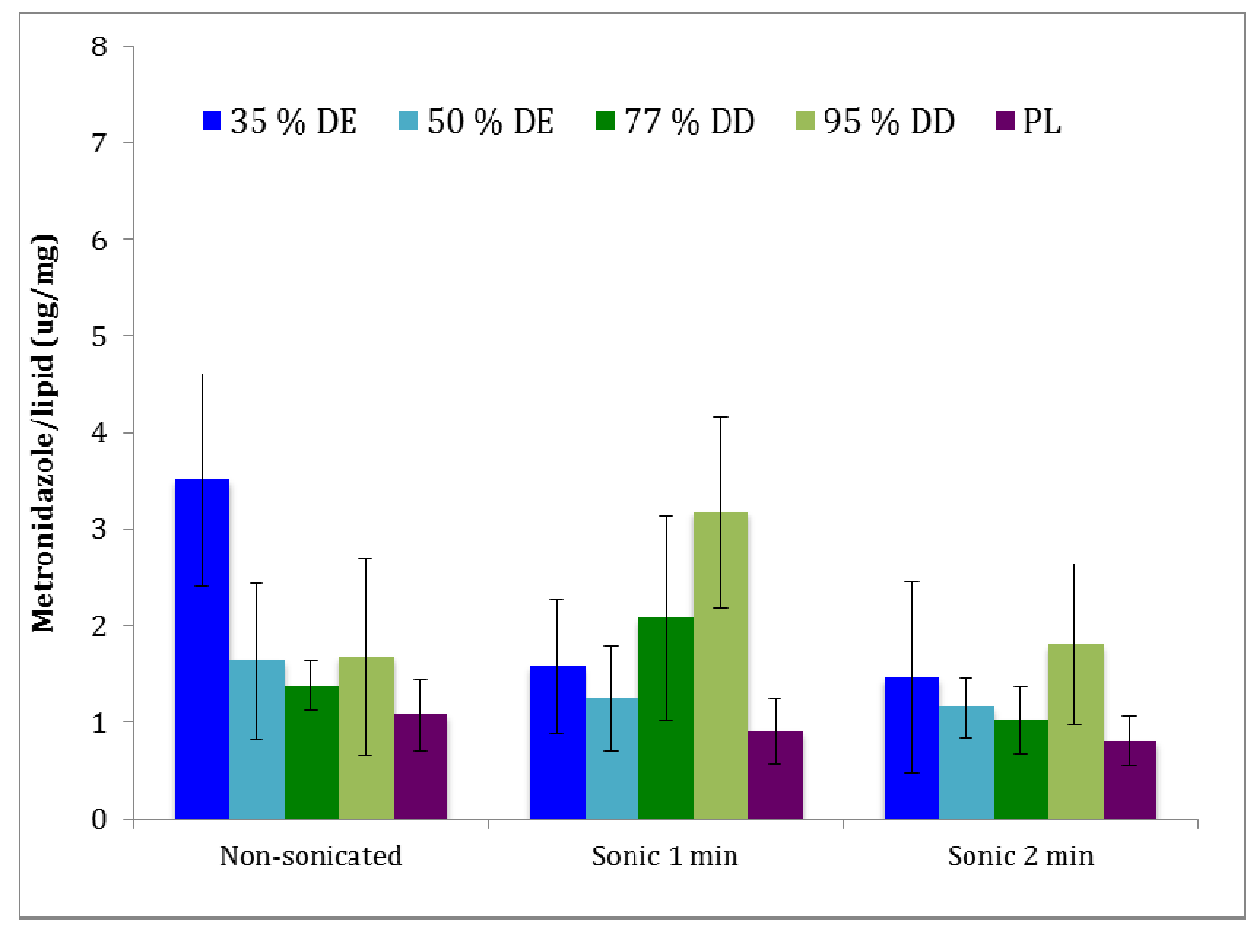

The potential problem of instability of newly developed vesicles upon storage in the cold conditions requires further testing at various temperatures and should be evaluated for the drugs of different lipophilicities.

\section{Conclusions}

The preparation process reported herein is simple and straightforward and has a potential to be used in the manufacturing of liposomes. The method can be applied for various types of polymers and possibly also polymer combinations.

Moreover, the method reduces the time required to manufacture mucoadhesive formulation. The newly developed vesicles were able to incorporate the sufficient amount of model antimicrobial agent. We are extensively characterizing the structure of the delivery systems contained in these formulations and trying to use the same approach to develop mucoadhesive nanopharmaceuticals for different antimicrobials interesting for vaginal administration. The real potential of the new type of vesicles remains to be confirmed in suitable in vitro and in vivo model.

\section{Acknowledgments}

T. Andersen is a PhD student jointly sponsored by the University of Tromsø, Norway and Umeå University, Sweden. The continuous generosity of Lipoid, Germany, and Herbstreith \& Fox, Germany, in providing the lipids and pectin, respectively, is highly appreciated. 


\section{Conflicts of Interest}

The authors declare no conflict of interest.

\section{References}

1. Hainer, B.L.; Gibson, M.V. Vaginitis: Diagnosis and treatment. Am. Fam. Physician 2011, 83, 807-815.

2. Vanić, Ž.; Škalko-Basnet, N. Nanopharmaceuticals for improved topical vaginal delivery: Can they deliver? Eur. J. Pharm. Sci. 2013, 50, 29-41.

3. Das Neves, J.; Amiji, M.; Sarmento, B. Mucoadhesive nanosystems for vaginal microbicide development: Friend or foe? Wiley Interdiscip. Rev. Nanomed. Nanobiotechnol. 2011, 3, 389-399.

4. Valenta, C. The use of mucoadhesive polymers in vaginal delivery. Adv. Drug Deliver. Rev. 2005, 57, 1692-1712.

5. Bernkop-Schnürch, A.; Dünnhaupt, S. Chitosan-based drug delivery systems. Eur. J. Pharm. Biopharm. 2012, 81, 463-469.

6. Hagesaether, E.; Sande, S.A. In vitro measurements of mucoadhesive properties of six types of pectin. Drug Dev. Ind. Pharm. 2007, 33, 417-425.

7. Sriamornsak, P. Application of pectin in oral drug delivery. Expert Opin. Drug Deliv. 2011, 8, 1009-1023.

8. Klemetsrud, T.; Jonassen, H.; Hiorth, M.; Kjøniksen, A.-L.; Smistad, G. Studies on pectin-coated liposomes and their interaction with mucin. Colloids Surf. B Biointerfaces 2013, 103, 158-165.

9. Ogończyk, D.; Siek, M.; Garstecki, P. Microfluidic formulation of pectin microbeads for encapsulation and controlled release of nanoparticles. Biomicrofluidics 2011, 5, 013405:1-013405:12.

10. Sharma, R.; Ahujaa, M.; Kaur, H. Thiolated pectin nanoparticles: Preparation, characterization and ex vivo corneal permeation study. Carbohydr. Polym. 2012. 87, 1606-1610.

11. Burapapadha, K.; Kumpugdee-Vollrathc, M.; Chantasartd, D.; Sriamornsak, P. Fabrication of pectin-based nanoemulsions loaded with itraconazole for pharmaceutical application. Carbohydr. Polym. 2010, 82, 384-393.

12. Nguyen, S.; Alund, S.J.; Hiorth, M.; Kjøniksen, A.-L.; Smistad, G. Studies on pectin coating of liposomes for drug delivery. Colloids Surf. B Biointerfaces 2011, 88, 664-673.

13. Karn, P.R.; Vanić, Ž.; Pepić, I.; Škalko-Basnet, N. Mucoadhesive liposomal delivery systems: The choice of coating material. Drug Dev. Ind. Pharm. 2011, 37, 482-488.

14. Takeuchi, H.; Yamamoto, H.; Niwa, T.; Hino, T.; Kawashima, Y. Mucoadhesion of polymer-coated liposomes to rat intestine in vitro. Chem. Pharm. Bull. 1994, 42, 1954-1956.

15. Manconi, M.; Mura, S.; Manca, M.L.; Fadda, A.M.; Dolz, M.; Hernandez, M.J.; Casanovas, A.; Díez-Sales, O. Chitosomes as drug delivery systems for C-phycocyanin: Preparation and characterization. Int. J. Pharm. 2010, 392, 92-100.

16. Vanić, Ž.; Hafner, A.; Bego, M.; Škalko-Basnet, N. The characterization of various deformable liposomes with metronidazole. Drug Dev. Ind. Pharm. 2013, 39, 481-488. 
17. Gentine, P.; Bubel, A.; Crucifix, C.; Bourel-Bonnet, L.; Frisch, B. Manufacture of liposomes by isopropanol injection: Characterisation of the method. J. Liposome Res. 2012, 22, 18-30.

18. Bartlett, G.R. Phosphorus assay in column chromatography. J. Biol. Chem. 1959, 234, 466-468.

19. Škalko, N.; Čajkovac, M.; Jalšenjak, I. Liposomes with metronidazole for topical use: The choice of preparation method and vehicle. J. Liposome Res. 1998, 8, 283-293.

20. Di Cagno, M.; Styskala, J.; Hlaváč, J.; Brandl, M.; Bauer-Brandl, A.; Škalko-Basnet, N. Liposomal solubilization of new 3-hydroxy-quinolinone derivatives with promising anticancer activity: A screening method to identify maximum incorporation capacity. J. Liposome Res. 2011, 21, 272-278.

21. Basnet, P.; Hussain, H.; Tho, I.; Škalko-Basnet, N. Liposomal delivery system enhances anti-inflammatory properties of curcumin. J. Pharm. Sci. 2012, 101, 598-609.

(C) 2013 by the authors; licensee MDPI, Basel, Switzerland. This article is an open access article distributed under the terms and conditions of the Creative Commons Attribution license (http://creativecommons.org/licenses/by/3.0/). 